Systematic Review

\title{
Home-Based Exercise Training for Heart Failure Patients
}

\section{Roby Aji Permana, Wikan Purwihantoro Sudarmaji, Wahyu Sukma Samudera, Agostinha Soares and Yanuar Aga Nugraha}

Faculty of Nursing, Universitas Airlangga, Surabaya, Indonesia

\begin{abstract}
Introduction: Exercise training is one of the recommendations for a cardiac rehabilitation program to increase exercise capacity and thus quality of life, decreasing both readmission and mortality in terms of heart failure. In spite of the evidence on the benefits and safety of cardiac rehabilitation, the uptake and participation of cardiac rehabilitation by patients with heart failure is currently poor. This study aimed to systematically review the effect of homebased exercise training on heart failure patients.
\end{abstract}

Methods: The studies were systematically identified by searching through the chosen electronic databases (Scopus, Science Direct, Proquest, Pubmed, and CINAHL) for articles from the 5 last years. The search algorithm identified a total 164 articles and 15 articles were selected based on the inclusion and exclusion criteria. For the 15 articles, 13 were RCTs, 1 was quasi-experimental and 1 was a retrospective study

Results: The major result of this review shows that home-based exercise has an effect on functional and exercise capacity, quality of life and a decreased rate of readmission within 2-12 months follow up

Conclusion: Home-based exercise has a long-term effect that is more effective than exercise that is hospital-based

\section{ARTICLE HISTORY}

Received: December 26, 2019

Accepted: December 31, 2019

\section{KEYWORDS}

home-based exercise; heart failure; blood pressure; hypertension

\section{CONTACT}

Roby Aji Permana

$\triangle$ roby.aji.permana-2018@fkp.unair.

$\equiv$ Faculty of Nursing, Universitas

Airlangga, Surabaya, Indonesia

Cite this as: Permana, R. A., Sudarmaji, W. P., Samudera, W. S., Soares, A., \& Nugraha, Y. A. (2019). Home-Based Exercise Training for Heart Failure Patients. Jurnal Ners, 14(3si), 155-160. doi:http://dx.doi.org/10.20473/jn.v14i3(si).16954

\section{INTRODUCTION}

Heart failure (HF) is an increasingly common cardiovascular syndrome and it is the final pathway in several cardiac disorders. Although mortality due to cardiovascular disease is decreasing, it remains one of the leading causes of death worldwide, placing a large social and economic burden on society. Many HF patients experience dyspnea, fatigue, diminished exercise capacity and poor quality of life(Kim et al., 2017; Kraal, Peek, Van Den Akker-Van Marle, \& Kemps, 2014).

Advances in pharmacological therapies and devices have been shown to improve the physiological parameters and quality of life, to reduce symptoms and to decrease mortality and the readmission rate. However, HF continues to have a significant negative impact on the quality of life of patients and their families or caregivers(Taylor et al., 2015). Exercise training is one of the recommendations for a cardiac rehabilitation program to increase exercise capacity and quality of life and to decrease readmission and mortality in heart failure. Cardiac Rehabilitation (CR) is continuously needed for optimal management.

In $\mathrm{HF}$, the patients lack regular exercise training and self-imposed limitations related to exercise may play an important role. Targeted physical activity is a vital element of comprehensive HF management (Smolis-b et al., 2015). Exercise training is a widely accepted intervention for $\mathrm{HF}$ leading to marked health benefits. In acquired heart failure, numerous studies have established unequivocally that regular exercise is related to better long-term prognosis and a reduced risk of cardiovascular death (Dissel et al., 2018). Research supports the importance of promoting exercise training to reduce mortality, hospitalization and the risk of other chronic diseases in patients with heart failure(Evangelista \& Cacciata, 2017).

Despite the benefits of exercise training programs, many patients do not have access to hospital- based 
training. The uptake and participation of cardiac rehabilitation by patients with heart failure is currently poor due to the knowledge of the program, distance, cost and poor health. One of the main reasons that people give for not accepting the invitation to attend cardiac rehabilitation are difficulties in regularly attending the sessions at their local hospital and a reluctance to take part in groupbased classes. Furthermore, the patients have a lack of knowledge on how they can improve or maintain the functioning of their body after they had heart failure(Peng et al., 2018).

Most studies on exercise in HF patients indicates the inability to access a formal rehabilitation program because they live in remote geographic locations, because they have difficulty with transportation or because do not have the financial resources to pay for a structured rehabilitation program. Recently, homebased exercise training programs have been introduced to increase the access and thus to reach a broader range of patients. It is anticipated that homebased exercise programs enhance patient selfefficacy and facilitate the lifelong implementation of regular physical activity with increased adherence, since it takes into consideration the preference of the individual patient (Dissel et al., 2018). Two keys solutions to this poor provision and uptake include the development of a home-based self-help CR manual designed to meet the needs of those with $\mathrm{HF}$ and the close involvement of their caregivers(Taylor et al., 2015). Currently, limited data is available on exercise prescription regarding safety, feasibility, and efficacy. To address this problem, home-based exercise training may be an acceptable alternative method for patients. This systematic review will give an overview of the effect of home-based exercise in compliance with the primary outcome of functional capacity and quality of life.

This systematic review sought to determine the effectiveness of home-based exercise programs compared with supervised centre-based exercise programs on exercise and/or the functional capacity, health-related quality of life, modifiable cardiac risk factors, mortality and morbidity of the patients with heart failure.

\section{MATERIALS AND METHODS}

A three step strategy was used for the collection of the literature study materials. The initial phase was conducted by searching the literature using a systematic review format on Chocrane, but a suitable theme was not found. The second step includes the keywords according to the selected topics in a selection of electronic databases: Scopus, PubMed, CINAHL, ScienceDirect and ProQuest. The articles were identified by the use of search terms and keywords ("home-based exercise", "heart failure", "functional capacity", "exercise capacity", "quality of life") published in the last 5 years (2015 - 2019) in English (Figure 1). Boolean logic was used for this searching strategy. The types of the studies that we selected were observational and experimental.

The titles and abstracts of the studies that were identified were screened and any clearly irrelevant studies discarded. The full-text of all of the potentially relevant studies were obtained and assessed independently for eligibility, based on the defined inclusion criteria. In cases where insufficient data was reported (e.g. method of randomization, statistical methods), this was discussed by the author and the risk of bias in the eligible studies was assessed in terms of the randomization and description of dropout and withdrawal.

The compilation of the review system followed the guidelines of the literature obtained from Cochrane. The PICOT framework used to formulate the answerable questions for this study. Population: the study population consisted of adult heart failure patients. Intervention: the intervention was homebased exercise. Comparison: usual care (center-based exercise). Output: exercise or functional capacity, quality of life, mortality and morbidity and readmission. The study population included adults with heart failure that had undergone revascularization through devices or patients with heart failure who had taken part, or been invited to take part, in cardiac rehabilitation.

Home-based exercise is defined as a structured program with clear objectives for the participants, including monitoring, follow-up visits and letters or telephone calls from the staff. The comparison group consisted of centre-based cardiac rehabilitation in a variety of settings (e.g. the hospital physiotherapy department, university gymnasium, community sports centre).

The primary outcome of this article is functional and exercise capacity and health-related quality of life. Mortality, morbidity and the readmission rate were included as a secondary outcome.

\section{RESULTS}

\section{Literature Search and Study Design}

A three-step strategy was used in the initial phase of the literature search in some databases with the specified keywords, which obtained 164 articles. The search found 14 articles from Scopus, 9 articles from PubMed, 15 articles from CINAHL, 79 articles from Proquest and 47 articles from ScienceDirect. The titles and authors of the studies were identified and duplicate studies were discarded. The second stage reviewed the abstracts retrieved according to the eligible criteria. We excluded the articles that did not match the inclusion criteria. The third step was reviewing the full articles. The full articles were reviewed using the PICOT framework.

The relevant data regarding the inclusion criteria (participants, interventions and outcomes), the risk of bias and the results was extracted. Data extraction was carried out by a single reviewer (RAP) and this was checked by a second reviewer (WPS). A total of 


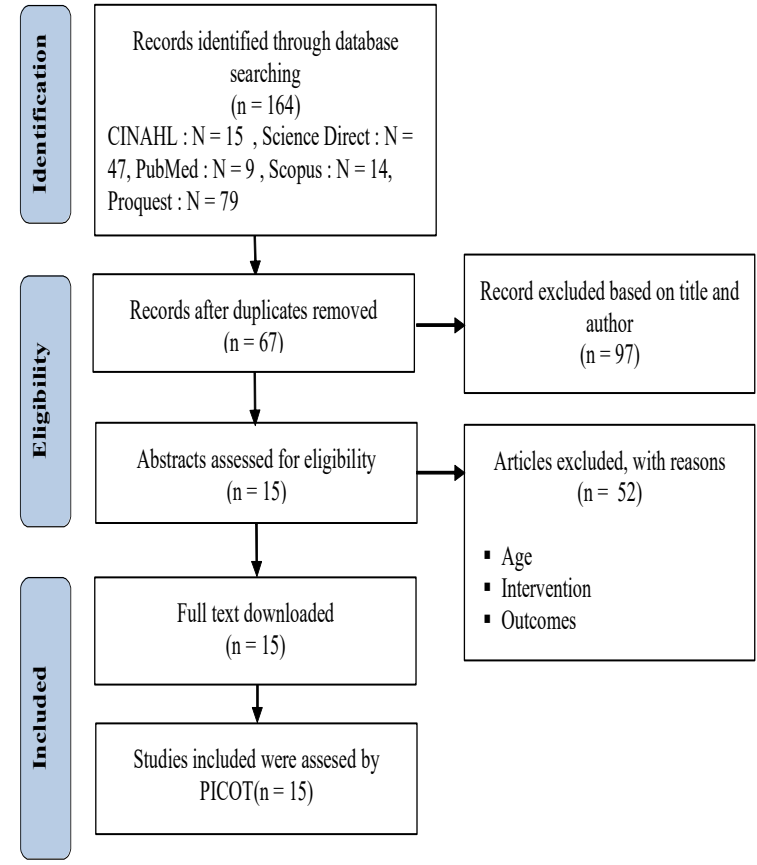

Figure 1. Prisma study flow diagram

15 articles were selected based on the criteria. The 15 articles included 13 RCTs, 1 quasi-experimental study and 1 retrospective study. The importance of the results of the study has been shown in Table 1 . Although a number of studies reported dropout rates, the reasons for the drop out were often unclear so it was therefore not possible to consistently estimate the number of patients withdrawing from the cardiac rehabilitation program for each study.

\section{Population}

The total number of respondents in this review was 5024. The population involved was between 16 to 3488 participants. The highest population was 3488 for the retrospective study and 360 from the randomized controlled trial. The participants involved were limited by several criteria such as age, clinical examination before action and their experience related to medical actions.

All of the studies were conducted on adults (aged 18 - 70 years). The criteria of the respondents in several studies was based on the classification of the New York Heart Association (stages 1 - 4) (Antonicelli et al., 2016; Evangelista \& Cacciata, 2017; George et al., 2017; Kim et al., 2017). Other criteria in the study were the fraction ejection, any co-morbidities and the stability status of the heart failure patients(Evangelista \& Cacciata, 2017; Kim et al., 2017; Nielsen, Duncan, \& Pozehl, 2018). The articles in this review came from 3 continents, namely America, Europe and Asia..

\section{Intervention Characteristic}

The intervention given to the respondents consisted of home-based physical activity/training. All of the studies used various models that were given to the patients. The home-based exercise interventions included walking exercises (Babu, Desai, Maiya,
Guddattu, \& Padmakumar, 2016; Hinrichs et al., 2016; Nielsen et al., 2018; Peng et al., 2018; Ramadi et al., 2015; Reeves, Whellan, Duncan, Connor, \& Pastva, 2017; Safiyari-hafizi, Taunton, Ignaszewski, \& Warburton, 2017; Shoemaker et al., 2017; Taylor et al., 2015), aerobic/strengthening exercises (Evangelista \& Cacciata, 2017; Hinrichs et al., 2016; Reeves et al., 2017; Shoemaker et al., 2017; Smolis-b et al., 2015), treadmill (Kim et al., 2017; Shoemaker et al., 2017), cycling/riding (Antonicelli et al., 2016; Evangelista \& Cacciata, 2017; Kim et al., 2017; Shoemaker et al., 2017) and self-selected exercise training(Dissel et al., 2018). Furthermore, some studies showed the adherence strategies for the home-based exercise over the duration of the study. Adherence strategies (logs, graphs, pedometers, phone follow-up, education, and a letter from CR staff) are intended to increase the rate of adherence(Nielsen et al., 2018). Some studies used telemonitoring/telehealth to follow-up as the strategy for better adherence.(Peng et al., 2018; Smolis-b et al., 2015). In addition, 1 study used the QQ and WeChat software to deliver the home-based telehealth exercise training(Peng et al., 2018).

The exercise period involved several duration differences; 2 months(Babu et al., 2016; Peng et al., 2018; Smolis-b et al., 2015), 3 months (Hinrichs et al., 2016; Kim et al., 2017; Nielsen et al., 2018; Ramadi et al., 2015; Reeves et al., 2017; Safiyari-hafizi et al., 2017; Shoemaker et al., 2017) and 6 months (Antonicelli et al., 2016; Dissel et al., 2018; Evangelista \& Cacciata, 2017; George et al., 2017).The frequency of exercise that had been done by the patient ranged between 2 - 6 sessions every week for 20 - 60 minutes/session. All reported outcomes at the follow up which instead reported change in the outcomes at follow up (3 - 24 months) compared to the baseline (Antonicelli et al., 2016; Peng et al., 2018; Ramadi et al., 2015).

\section{Clinical Outcome}

A number of studies give a specific primary outcome. The primary outcome in most of the studies was functional capacity/exercise capacity, quality of life, mortality and morbidity and the rate of readmission. Functional capacity was measured by a 6-minute walking test. Exercise capacity is measured as the maximal oxygen uptake (V02max), referring to either the metabolic equivalents (METs) or milliliters per kilogram of body mass per minute $(\mathrm{ml} / \mathrm{kg} / \mathrm{min})$ or milliliters (ml)(Safiyari-hafizi et al., 2017; Smolis-b et al., 2015).

Quality of life was measured by the Minessotta Living with Heart Failure Questionnaire (MLHFQ) and the medical outcome Survey-short Form 36SF36(Babu et al., 2016). Changes in the condition were associated with readmission rate, mortality and morbidity, which were also documented in the studies with the maximum follow up being up to 12 months (Antonicelli et al., 2016; Peng et al., 2018; Ramadi et al., 2015). 


\section{DISCUSSION}

Exercise training is a well-established adjunct therapy in acquired heart failure. Substantial evidence shows that exercise markedly affects the prognosis and reduces mortality and hospitalization. It also improves functional capacity, exercise capacity and health-related quality of life (Dissel et al., 2018). The chance of most patients with HF improving their exercise tolerance was limited due to an increased risk of related adverse events, frequently advanced age, poor adherence and comorbidities. In spite of that, attempts to promote regular physical activity such as diverse exercise training, are independent and effective options to improve the patients' health status(Smolis-b et al., 2015).

Increased functional capacity occurs due to the improved muscle pump function caused by many factors, including medical therapy, lifestyle education and exercise activity(McKelvie, 2008). Smolis (2015) showed that after a period of home -based exercise, there were beneficial effects in terms of left ventricular remodeling and the parameters of exercise capacity in the cardiopulmonary exercise testing and 6MWT could be observed with a greater improvement seen in the patients participating in the regular exercise training program(Smolis-b et al., 2015).

Exercise in heart failure facilitates the physiological adaptation of muscles that are trained to increase oxygen uptake, reduce oxidative stress, increase enzyme aerobics and increase the number of muscle type I(McKelvie, 2008). The findings of Ramadi et al (2015) showed that every $1 \mathrm{~mL} \cdot \mathrm{kg}-1$ - min - 1 (ie, $0.28 \mathrm{METs}$ ) increase in exercise capacity is associated with a $10 \%$ reduction in cardiovascular mortality. Increases of 0.95 METs and 0.73 METs in exercise capacity (for the center-based and homebased groups, respectively) indicate the potential clinical significance of this study(Ramadi et al., 2015). The significant improvement in aerobic power is important for improving the functional reserve of the patients with $\mathrm{HF}$ and reducing the risk for disability/functional dependence and premature mortality (Safiyari-hafizi et al., 2017).

Physical exercise will stimulate the vasodilating factor of the vascular endothelium that improves remodeling in vascularization. This condition contributes to a decrease in peripheral vascular resistance, an increase $\mathrm{n}$ ejection fraction and improved stroke volume(McKelvie, 2008).The major new finding from Safiyari-hafizi (2017) was that supervised home-based cardiac rehabilitation included a combination of high intensity interval and resistance training which resulted in a significant improvement in the VO2 peak in patients with HF. Furthermore, the study showed a significant improvement in exercise capacity, quality of life, and the oxygen consumption at the ventilatory threshold (Safiyari-hafizi et al., 2017).

Rehabilitation exercise can minimize the symptoms, increase exercise tolerance and quality of life and have a satisfying effect on patient recovery. Home-based exercise was proven to increase exercise capacity and self efficacy and decrease the rate of readmission (McKelvie, 2008). Exercise training has significant implications for the health status and overall quality of life of $\mathrm{HF}$ patients. Given the important role that aerobic power and functional status plays in morbidity and mortality in HF, exercise training may also have potentially important implications for improving survival in $\mathrm{HF}$ and reducing the hospitalizations and the health care costs associated with treating HF. Safiyari-Hafizi (2017) revealed that their closely monitored homebased cardiac rehabilitation program (employing high intensity interval and resistance training) resulted in significant improvements in physiological well-being and overall quality of life. This is consistent with the other trials of HF management that support the use of home-based aerobic training programs that include close monitoring and interactions with the patients via a follow up. This speaks directly to the applicability and efficacy of home-based interval training in persons living with HF(Safiyari-hafizi et al., 2017).

Prescribing physical exercise, adjusting the physical prescriptions and integrating exercises affect patient compliance when doing exercises. Prescribing an adequate level of physical exercise in patients with heart failure should include the components of frequency, intensity, duration and the physical exercise mode. Physical exercise adjustment prescriptions are needed to get optimal physical exercise results. An integrated training program should start when the patient is stable, followed by focused physical exercise in the hospital. After the patient returns home, they should continue independently with a home-based exercise. Patients who start home-based rehabilitation programs must be convinced in terms of that their knowledge about physical training, their continuous access to the monitoring center and the telemonitoring guidance in that it guarantees their absolute safety. It is also important to motivate the patients to continue exercise training in all stages of rehabilitation and to offer them comprehensive care to maintain good exercise tolerance(Smolis-b et al., 2015).

In home-based programs, there is more emphasis on self-monitoring and independent exercise. Selfmonitoring might in turn enhance the patient awareness of their behavior, which could ultimately lead to better adherence to long-term behavior changes. Furthermore, developing an early adaptation of exercise behavior changes in the patient's home environment may play a key role in exercise sustainability (Ramadi et al., 2015). Homebased exercise programs help to address these issues by enabling the elderly to exercise in the comfort of their own home using a program that was developed to be safe and suitable for elderly $\mathrm{HF}$ patients. Additionally, giving the participants better access to an in home exercise program, as well as following up with regular phone calls from the study physician, 
may have led to the increase in the positivity of their perceived physical and mental health, as indicated by the change in the SF-36 scores. This increase in positive health perception might have led to the perception of increased energy, reduced fatigue, increased health and a reduction in the limitations that their physical issues placed on their normal life activities in the intervention group (George et al., 2017).

A structured home-based program must be prescribed clearly, including its objective, duration, intensity, adherence strategy and the monitoring or follow up visits. There are many confounding factors that will affect the program such as the magnitude of inactivity, the duration, environment and the customization of the exercise intervention. The most significant confounding factor is the readiness for behavioral change. In several studies, it is unknown the degree to which the subjects included in the study were ready for behavioral change (Shoemaker et al., 2017). The initiation of behavioral change is needed to improve awareness and to increase the knowledge that will provide compliance in the patients included in the rehabilitation program. The various patients included in the rehabilitation program showed that home-based cardiac rehabilitation can be an alternative or additional program for patients with heart failure.

\section{CONCLUSION}

The major result of review shows that home based exercise has an effect on functional and exercise capacity, quality of life, and decrease the rate of readmission within the $2-12$ months of the follow up. Home-based exercise has a long-term effect that is more effective than hospital based. Furthermore, a structured home based exercise program must be considered according to the duration, environment, customization and the readiness for behavioral change in the patients with heart failure.

\section{REFERENCES}

Antonicelli, R., Spazzafumo, L., Scalvini, S., Olivieri, F., Mattasini, M. V., Parati, G., ... Lattanzio, F. (2016). Exercise: A "New Drug" for Elderly Patients With Chronic Heart Failure. Aging, 8(5), 860-869.

Babu, A. S., Desai, C. V., Maiya, A. G., Guddattu, V., \& Padmakumar, R. (2016). Changes in derived measures from six-minute walk distance following home-based exercise training in congestive heart failure: A preliminary report. Indian Heart Journal, 68, 527-528. https://doi.org/10.1016/j.ihj.2016.05.010

Dissel, A. C. Van, Hooglugt, J. Q., De, F. H., Blok, I. M., Jørstad, H. T., Mulder, B. J. M., ... Winter, M. M. (2018). Safety and Effectiveness of Home-Based, Self-Selected Exercise Training in Symptomatic Adults with Congenital Heart Disease: A Prospective, Randomised, Controlled Trial.
International Journal of Cardiology. https://doi.org/10.1016/j.ijcard.2018.12.042

Evangelista, L. S., \& Cacciata, M. (2017). DoseResponse Relationship Between Exercise Intensity, Mood States, and Quality of Life in Patients With Heart Failure. Journal of Cardiovascular Nursing, 1-8. https://doi.org/10.1097/JCN.000000000000040 7

George, M., Azhar, G., Pangle, A., Peeler, E., Dawson, A., Coker, R., ... Wei, J. (2017). Feasibility of Conducting a 6-Months Long Home-based Exercise Program with Protein Supplementation in Elderly Community-dwelling Individuals with Heart Failure. Journal of Physiotherapy \& Physical Rehabilitation, 2(2), 19-22. https://doi.org/10.4172/2573-0312.1000137

Hinrichs, T., Bucker, B., Klaaßen-mielke, R., Brach, M., Dipl-Stat, Wilm, S., ... Mai, A. (2016). Home-Based Exercise Supported by General Practitioner Practices: Ineffective in a Sample of Chronically Ill, Mobility-Limited Older Adults (The HOMEfit Randomized Controlled Trial). The American Geriatrics Society, 1-10. https://doi.org/10.1111/jgs.14392

Kim, M., Kim, M. S., Lim, S. J., Ahn, J. M., Kim, J. J., \& Park, S. J. (2017). Comparison of supervised hospitalbased versus educated home-based exercise training in Korean heart failure patients. Korean Circulation Journal, 47, 742-751. https://doi.org/10.4070/kcj.2017.0061

Kraal, J. J., Peek, N., Van Den Akker-Van Marle, M. E., \& Kemps, H. mc. (2014). Effects of home-based training with telemonitoring guidance in low to moderate risk patients entering cardiac rehabilitation: Short-term results of the FIT@Home study. European Journal of Preventive Cardiology, 21, 26-31. https://doi.org/10.1177/2047487314552606

McKelvie, R. S. (2008). Exercise training in patients with heart failure: Clinical outcomes, safety, and indications. Heart Failure Reviews, 13(1), 3-11. https://doi.org/10.1007/s10741-007-9052-z

Nielsen, J., Duncan, K., \& Pozehl, B. (2018). PatientSelected Strategies for Post Cardiac Rehabilitation Exercise Adherence in Heart Failure. Rehabilitation Nursing Journal, 1-5. https://doi.org/10.1097/rnj.000000000000012 7

Peng, X., Su, Y., Hu, Z., Sun, X., Li, X., Dolansky, M. A., ... $\mathrm{Hu}, \mathrm{X}$. (2018). Home-based telehealth exercise training program in Chinese patients with heart failure. Medicine, 97(August), 1-9.

Ramadi, A., Haennel, R. G., Stone, J. A., Arena, R., 
Threlfall, T. G., Hitt, E., ... Martin, B. (2015). The Sustainability of Exercise Capacity Changes in Home Versus Center-Based Cardiac Rehabilitation. Journal of Cardiopulmonary Rabilitation and Prevention, 35, 21-28. https://doi.org/10.1097/HCR.00000000000000 84

Reeves, G. R., Whellan, D. J., Duncan, P., Connor, M. O., \& Pastva, A. M. (2017). Rehabilitation Therapy in Older Acute Heart Failure Patients ( REHAB-HF ) trial: Design and rationale. American Heart Journal, 185, 130-139. https://doi.org/10.1016/j.ahj.2016.12.012

Safiyari-hafizi, H., Taunton, J., Ignaszewski, A., \& Warburton, D. (2017). The Health Benefits of a 12 Week Home-Based Interval Training Cardiac Rehabilitation Program in Patients with Heart Failure. Canadian Journal of Cardiology, (2016). https://doi.org/10.1016/j.cjca.2016.01.031

Shoemaker, M. J., Oberholtzer, N. L., Jongekrijg, L. E., Bowen, T. E., Cartwright, K., Hanson, K., ... Kowalk, A. (2017). Exercise- and Psychosocial-Based
Interventions to Improve Daily Activity in Heart Failure: A Pilot Study. Home Health Care Management \& Practice, 1-10. https://doi.org/10.1177/1084822316683660

Smolis-b, E., D, R., Piotrowicz, E., Chwyczko, T., Dobraszkiewicz-wasilewska, B., Kowalik, I., ... Szwed, H. (2015). Hospital-based and telemonitoring guided home-based training programs: Effects on exercise tolerance and quality of life in patients with heart failure (NYHA class III ) and cardiac resynchronization therapy . A randomized, prospective observation. International Journal of Cardiology, 199, 442-447. https://doi.org/10.1016/j.ijcard.2015.07.041

Taylor, R. S., Hayward, C., Eyre, V., Austin, J., Davies, R., Doherty, P., ... Britten, N. (2015). Clinical effectiveness and cost- effectiveness of the Rehabilitation Enablement in Chronic Heart Failure rehabilitation intervention in heart failure patients and caregivers: rationale and protocol for a multicentre randomised controlled trial. $B M J$ Journal, 5(12), 1-11. https://doi.org/10.1136/bmjopen-2015-009994 